\title{
Health Information Systems Is our Focus Correct?
}

\author{
C. Bréant, Section Editor for the IMIA Yearbook Section on Health Information Systems \\ Department of Imaging and Medical Informatics, Geneva University Hospitals, Geneva, Switzerland
}

\section{Introduction}

HIS implementation and use increase at a steady pace therefore building some evidence that information technology has a legitimate place in the transformation of health care and the improvement of patient outcome. The design of HIS relies on complex architectures and technologies which have reached significant levels of maturity after several decades of development [1,2]. Still, the rate of adoption of HIS is low, hindered by many barriers such as costs and interoperability. Additionally, perception that these systems don't adequately address all the complexity of health care and clinical work issues slows HIS deployment [3].

Current publications indicate that there is a need for fundamental research on human factors engineering. Indeed, it appears that organizational and human challenges are more difficult to master than technical aspects. This clearly demonstrates that within HIS projects and implementations, the focus has shifted from pure technical issues to organizational and non-functional aspects [4-11].

It is worth noting that HIS implementations are large and costly projects. It requires difficult strategic choices at the management level coupled with numerous organizational skills. Moreover, in order to justify large financial investments, value of HIS must be demonstrated to stakeholders [10]. Meta analyses of a large amount of studies performed on these topics are helping to find answers to these fundamental questions and to capitalize on work already performed by a large panel of HIS professionals $[8,10]$.

\section{About the Paper Selection}

The selection of papers for the section on Health Information Systems in the IMIA Yearbook 2011 is based on a thorough review of published articles that best address today's challenges of HIS deployment and implementation $[13,14]$. Table 1 lists the five selected papers from international peer reviewed journals in the fields of medicine and medical informatics. A brief content summary of the five selected paper can be found in the appendix of this synopsis.

\section{Conclusion and Outlook}

This year papers highlight the multidimensional aspects involved in the deployment of a HIS system. These can be analyzed from large corpuses of published studies $[4,8,10]$.

As described in the first paper selected, national electronic health records programs usually encounter many problems due to their costs, size, and long duration. The analysis of these programs in five industrialized countries shows that despite having faced similar problems, the different countries have 
looked for individual solutions. Future important organizational, strategic, and human challenges could benefit from a comprehensive method to support the implementation of such programs [9].

Health information exchange (HIE) applications is the focus of the second paper. These are crucial components to the sharing of health data in a fragmented national health care setting. The need to be able to evaluate their respective costs, effort, and value has led to the design of a framework defining all useful measures and metrics. The results should help future choices of strategic investments and plans to increase the chances of a successful adoption [10].

As shown in the third paper, the adherence of electronic health records to rigid quality requirements is necessary in order to support and improve health care. A systematic review of a large set of studies outlines the importance of non functional requirements such as portability, performance and maintenance issues [4].

The fourth paper reports that evidence of the effects of HIS on the improvement of quality of care can be demonstrated from the consolidation of the many studies published to date [8]. Key success factors can be singled out to help future implementations.

The last and fifth paper focuses on radical changes in the clinician workflow which are often a consequence of the introduction of information technology in a clinical setting. Clinician time might be redistributed, thus creating the perception of decreased clinical performance, user satisfaction, and quality of care. Innovative analytical methods based on workflow analysis can enrich traditional time utilization methods to improve the understanding of the impact introduced by information technology in clinical settings [3].

\section{Acknowledgement}

I greatly acknowledge the support of Martina Hutter and of the reviewers in the selection process of the IMIA Yearbook.

Table 1 Best paper selection of articles for the IMIA Yearbook of Medical Informatics 2011 in the section 'Health Information Systems'. The articles are listed in alphabetical order of the first author's surname.

\section{Section \\ Health Information Systems}

- Deutsch E, Duftschmid G, Dorda W. Critical areas of national electronic health record programs-is our focus correct? Int J Med Inform 2010 Mar;79(3):211-22. Epub 2010 Jan 15.

- Dixon BE, Zafar A, Overhage JM. A Framework for evaluating the costs, effort, and value of nationwide health information exchange. J Am Med Inform Assoc 2010 May 1;17(3):295-301.

- Hoerbst A, Ammenwerth E. Electronic health records. A systematic review on quality requirements. Methods Inf Med 2010;49(4):320-36. Epub 2010 Jul 6.

- Lau F, Kuziemsky C, Price M, Gardner J. A review on systematic reviews of health information system studies. J Am Med Inform Assoc 2010 Nov 1;17(6):637-45

- Zheng K, Haftel HM, Hirschl RB, O'Reilly M, Hanauer DA. Quantifying the impact of health IT implementations on clinical workflow: a new methodological perspective. J Am Med Inform Assoc 2010 Jul-Aug; 17(4):454-61.

\section{References}

1. Friedman DJ, Parrish RG. The population health record: concepts, definition, design, and implementation. J Am Med Inform Assoc 2010 Jul-Aug;17(4):359-66.

2. Arnrich B, Mayora O, Bardram J, Tröster G. Pervasive healthcare: paving the way for a pervasive, user-centered and preventive healthcare model. Methods Inf Med 2010;49(1):67-73. Epub 2009 Dec 8

3. Zheng K, Haftel HM, Hirschl RB, O'Reilly M, Hanauer DA. Quantifying the impact of health IT implementations on clinical workflow: a new methodological perspective. J Am Med Inform Assoc 2010 Jul-Aug;17(4):454-61.

4. Hoerbst A, Ammenwerth E. Electronic health records. A systematic review on quality requirements. Methods Inf Med 2010;49(4):32036. Epub 2010 Jul 6.

5. Karsh BT, Weinger MB, Abbott PA, Wears RL. Health information technology: fallacies and sober realities. J Am Med Inform Assoc 2010 Nov 1;17(6):617-23.

6. Bansler JP, Havn E. Pilot implementation of health information systems: issues and challenges. Int $\mathrm{J}$ Med Inform 2010 Sep;79(9):637-48. Epub 2010 Jun 23.

7. Vest JR. More than just a question of technology: factors related to hospitals' adoption and implementation of health information exchange. Int J Med Inform 2010 Dec; 79(12):797-806.

8. Lau F, Kuziemsky C, Price M, Gardner J. A review on systematic reviews of health information system studies. J Am Med Inform Assoc 2010 Nov 1;17(6):637-45.

9. Deutsch E, Duftschmid G, Dorda W. Critical areas of national electronic health record programs-is our focus correct? Int J Med Inform 2010 Mar;79(3):21122. Epub 2010 Jan 15.

10. Dixon BE, Zafar A, Overhage JM. A Framework for evaluating the costs, effort, and value of nationwide health information exchange. J Am Med Inform Assoc 2010 May 1;17(3):295-301
11. Maxson E, Jain S, Kendall M, Mostashari F Blumenthal D. The regional extension center program: helping physicians meaningfully use health information technology. Ann Intern Med 2010 Nov 16;153(10):666-70.

12. Hoerbst A, Kohl CD, Knaup P, Ammenwerth E. Attitudes and behaviors related to the introduction of electronic health records among Austrian and German citizens. Int J Med Inform 2010 Feb;79(2):81-9. Epub 2009 Dec 23.

13. Bréant C. Health Information Systems - New Shifts and Emerging Trends in Health IT Infrastructures. Yearb Med Inform 2009:71-2.

14. Bréant C. Health Information Systems. Understanding Health Care IT Alignment. Yearb Med Inform.2010:30-3.

Correspondence to:

Claudine Bréant, PhD

Direction de l'analyse médico-économique

Geneva University Hospitals

Rue Gabrielle-Perret-Gentil, 4

1211 Geneva, Switzerland

Tel: +41223726179

Fax: +41223728879

E-mail: claudine.breant@hcuge.ch

\section{Appendix: Content Summaries of Selected Best Papers for the IMIA Yearbook 2011, Section 'Health Information Systems*}

\footnotetext{
* The complete papers can be accessed in the Yearbook's full electronic version, provided that permission has been granted by the copyright holder(s).
} 


\section{Deutsch E, Duftschmid G, Dorda W Critical areas of national electronic health record programs-Is our focus correct? Int J Med Inform 2010 Mar ; 79(3):211-22. Epub 2010 Jan 15}

National electronic health record (EHR) programs are seen by many countries as an opportunity to improve the public health sector. However, initial implementations have demonstrated the complexity of such programs requiring many years of costly investments. The frequent problems reported include: the slow progress of the projects, their cost, the discussions over the choice for an implementation strategy, and the poor acceptance in the medical community. In this report, the most common critical aspects of national electronic health record programs are compiled from the analysis of the situation in five industrialized countries (Canada, Australia, England, Denmark, and Germany). The authors bring a new contribution to this topic by comparing implementation choices in five different countries. The study is based on project reviews and audits following the implementation of each program. Different levels of achievement can be observed: (a) programs for a national electronic health record system which exist since at least 5 years (b) planned electronic health record systems encompassing various approaches of implementation, and (c) pilot projects which already have been conducted. The critical areas frequently involved include: (a) acceptance and change management, (b) demonstration of benefits and funding, (c) project management, (d) health-policy-related goals and implementation strategy, (e) basic legal requirements, such as data protection. The analysis shows that similar critical areas exist in the various countries. One important message is that strategic, organizational and human challenges are usually more difficult to master than technical aspects. In conclusion, the authors suggest that a comprehensive method should be provided to support the complete process of implementing a national electronic health record program. It should cover all critical areas identified within this study. It is also suggested that this method should be enriched by successful measures implemented in individual countries.

\section{Dixon BE, Zafar A, Overhage JM A Framework for evaluating the costs, effort, and value of nationwide health information exchange \\ J Am Med Inform Assoc 2010 May $1 ; 17(3): 295-301$}

Implementing a nationwide health information network (NHIN) program requires the development of infrastructures facilitating the adoption of systems for the exchange of electronic data among disparate and competing healthcare institutions usually found in a fragmented healthcare delivery system. As such, the United States will soon put in production a NHIN to securely link community and state health information exchange (HIE) entities, thus enabling a national, interoperable network for sharing healthcare data throughout the country. The evaluation of such costly programs is important because it enables HIE organizations to demonstrate value to their stakeholders. However, the evaluation of NHIN, and the development of general frameworks and methods for evaluating HIE have been rarely studied and addressed in publications so far. Using the literature review and knowledge gained from the NHIN technology and policy development, the authors have constructed a framework for evaluating the costs, effort, and value of data exchange between a HIE entity and the NHIN. An online survey, addressed to HIE professionals and researchers, was used to collect data regarding the perceived usefulness of the metrics in the proposed framework. It is organized into five broad categories: implementation, technology, policy, data, and value.
Each category enumerates a variety of measures and measure types, which are detailed in the paper. Survey respondents indicated if the framework contained useful measures for current and future use in HIE and NHIN evaluations. In summary, nearly all of the metrics and category were rated as useful or very useful, with the value category standing out. In conclusion, the results of this study in an important contribution to the development of an evidence base for both helping with the adoption of HIE, and demonstrating the value of such systems.

\section{Hoerbst A, Ammenwerth E \\ Electronic health records. A systematic review on quality requirements}

\section{Methods Inf Med. 2010;49(4):320-36. Epub 2010 Jul 6}

The concept of electronic health records (EHRs) emerged in the 1990s. The content, structure, and technology of EHRs have evolved in times, but the basic ideas which are the quality of health care and supporting all stakeholders in the health care process have remained. It is however crucial that EHRs adhere to rigid quality requirements to meet challenges such as cost and interoperability in a highly competitive environment with heterogeneous requirements. This review presents a first attempt to identify mainly non-functional, quality requirements of electronic health records. It is based from an analysis of both published current literature and expert interviews. The presented study includes sources and experts from different domains such as standards and norms, scientific literature and guidelines, and best practice. As a result, more than 1200 requirements were identified of which 203 requirements were also mentioned during the expert interviews. An important contribution by the authors is the categorisation of these requirements. Categories with the highest number of requirements found include global functional requirements 
and data security. By comparison, the number of non-functional requirements found is lower. The authors suggest that the results of this study can be useful during the conceptual design or the refinement step of EHR systems. In conclusion, the authors highlight the fact that the systematic identification of a proven set of requirements for EHR systems is necessary to improve future EHRs implementations. Most importantly, it should include both functional as well as non-functional requirements.

Lau F, Kuziemsky C, Price M, Gardner J

A review on systematic reviews of health information system studies

\section{J Am Med Inform Assoc 2010 Nov $1 ; 17(6): 637-45$}

Despite an impressive number of studies along with their systematic reviews published in the last decades, evidence of the effects of HIS on quality of care is still a current debate with mixed or event contradictory results. The purpose of this meta-analysis is to consolidate and cumulate results from existing evidence from a large set of published systematic reviews. The results will give the current knowledge on the effects of HIS. Fifty reviews published during 1994-2008, corresponding to a non-overlapping corpus of 1276 HIS studies were selected for meta-level synthesis. The five areas covered by these reviews include: medication management, preventive care, health conditions, data quality, and care process/outcome. On the basis of a subset of 287 controlled HIS studies, there is some evidence for improved quality of care. Results vary across HIS features reported. Key factors that influence HIS success included having in-house systems, developers as users, integrated decision support and benchmark practices, and addressing such contextual issues as provider knowledge and perception, incentives, and legislation/policy. Furthermore, the result of this comprehensive study offers a guide on the work performed to date on the evaluation of HIS. It also provides useful recommendations and help to identify future research needs specially to increase HIS adoption.

\section{Zheng K, Haftel HM, Hirschl RB, O'Reilly M, Hanauer DA \\ Quantifying the impact of health IT implementations on clinical workflow: a new methodological perspective \\ J Am Med Inform Assoc 2010 Jul- Aug; 17(4):454-61}

Health IT implementations such as EHR and computer process order entry (CPOE) often introduce radical changes to clinical work processes, possibly impeding user satisfaction and quality of care. Indeed, prior research on this topic shows non-significant and even conflicting results. Recent time and motion studies have consistently found that the impact of health IT implementations is negligible; whereas qualitative studies have repeatedly revealed negative end-user perceptions of decreased efficiency and disrupted workflow. The author makes the hypothesis that this discrepancy might be due to the focus of these studies on measuring clinician's time expenditures among different clinical activities rather than analyzing clinical workflow from the real flow of the work perspective. This paper presents a new analytical method to better quantify health IT impact on workflow, thus improving current approaches. The new analytical method consists of workflow fragmentation assessments, pattern recognition, and data visualization, which are designed to uncover hidden regularities embedded in the flow of the work. An important contribution to this topic is the use of data visualization techniques to derive more comprehensive and analytic information from complex clinical workflows. The potential value of these new methods is demonstrated through an empirical study conducted in a 16 -bed pediatric intensive care unit, when a CPOE system was newly installed. Pre-implementation data and post-implementation data show that several task transitions had become much more frequent after the CPOE system has been implemented. An increase of task switching can account for a perceived decreased time efficiency and disrupted workflow; whereas a time utilization analysis doesn't suggest any impact. Although limited in size and scope, and therefore not directly usable in a general setting, this empirical study nevertheless shows that these new methods can contribute to explain and to uncover the paradox between quantitative and qualitative results in workflow analysis in clinical settings. 